\title{
Hipertireoidismo na gestação
}

\author{
Hyperthyroidism in Pregnancy
}

Hipertiroidismo en la gestación

Bárbara B.G. R. Bártholo, Denise L. M. Monteiro, ${ }^{*}$ Alexandre J. B. Trajano*

\section{Resumo}

O objetivo desta revisão é destacar que a identificação precoce do hipertireoidismo na gestação possibilita início precoce de tratamento, evitando graves consequências para a saúde materno-fetal. A prevalência de hipertireoidismo na gestação varia entre 0,1 a $0,4 \%$ e pode se manifestar clinicamente como hipertireoidismo subclínico, tireotoxicose e crise tireotóxica. O diagnóstico é suspeitado pela ocorrência de perda de peso, sudorese, ansiedade, taquicardia, hipertensão arterial e aumento do volume da tireoide, sendo confirmado por supressão ou não detecção dos níveis séricos de TSH e por elevação do T4L. O TRAb deverá ser solicitado quando a etiologia for desconhecida, nas pacientes submetidas a tratamento com iodo radioativo ou tireoidectomia por doença de Graves (DG) antes da gestação ou com achados clínicos compatíveis com a doença. As gestantes com hipertireoidismo devem ser acompanhadas com dosagem de TSH, T4L e T3L na primeira consulta de pré-natal e mensalmente. $\mathrm{O}$ tratamento do hipertireoidismo baseia-se no uso de propiltiouracil (PTU) e metimazol (MMZ). No primeiro trimestre de gestação deve-se usar o PTU devido aos efeitos do MMZ em nível embrionário. Entretanto, o PTU deverá ser substituído pelo MMZ após este período por sua hepatotoxicidade. Pacientes em uso de PTU devem ter as enzimas hepáticas monitoradas durante o tratamento a cada quatro semanas assim como a função tireoidiana. O diagnóstico precoce, a identificação da etiologia e o tratamento precoce e adequado do hipertireoidismo são importantes para evitar complicações como: crise tireotóxica, abortamento, hipertensão gestacional, baixo peso ao nascer, restrição de crescimento intrauterino, natimortalidade, disfunção tireoidiana neonatal, parto prematuro e descolamento prematuro de placenta.

Descritores: Hipertireoidismo; Gravidez; Tratamento.

\begin{abstract}
The aim of this review is to highlight that early identification of hyperthyroidism during pregnancy allows early initiation of treatment, avoiding serious consequences for maternal and fetal health. The prevalence of hyperthyroidism in pregnancy ranges from 0.1 to $0.4 \%$ and may manifest clinically as subclinical hyperthyroidism, thyrotoxicosis and thyroid storm. The diagnosis is suspected by the occurrence of weight loss, sweating, anxiety, tachycardia, hypertension and increased thyroid volume, being confirmed by deletion or non-detection of serum TSH and free T4 (FT4) elevation. The TRAb should be requested when the etiology is unknown, those undergoing treatment with radioactive iodine or thyroidectomy before pregnancy due to Graves' disease (GD) or clinical findings compatible with the disease. Pregnant women with hyperthyroidism should be accompanied with TSH, FT4 and FT3 at the first prenatal consultation and monthly. The treatment of hyperthyroidism is based on the use of propylthiouracil (PTU) and methimazole (MMZ). In the first trimester of pregnancy PTU should be used to from the effects of MMZ embryonic level. However, the PTU should be replaced by MMZ after this period for its hepatotoxicity. Patients using PTU should have liver enzymes monitored during treatment every four weeks as well as thyroid function. Early diagnosis and identification of the etiology and early and appropriate treatment of hyperthyroidism are important to avoid complications such as thyroid storm, miscarriage, gestational hypertension, low birth weight, intrauterine growth restriction, stillbirth, neonatal thyroid dysfunction, premature birth and placental abruption.
\end{abstract}

Keywords: Hyperthyroidism; Pregnancy; Treatment. 


\section{Resumen}

El objetivo de esta revisión es destacar que la identificación precoz del hipertiroidismo en la gestación posibilita el inicio adelantado del tratamiento, evitando graves consecuencias para la salud materno-infantil. La prevalencia de hipertiroidismo en la gestación varia entre el 0,1 al 0,4\% y puede manifestarse clínicamente como hipertiroidismo subclínico, tirotoxicosis y crisis tirotóxica. Se sospecha el diagnóstico por la ocurrencia de perdida de peso, sudores, ansiedad, taquicardia, hipertensión arterial y aumento del volumen de la tiroides, siendo confirmado por la supresión o no detección de los niveles séricos de TSH y por elevación del T4L. El TRAb deberá ser solicitado cuando la etiología fuese desconocida, en aquellas pacientes sometidas al tratamiento con yodo radioactivo o tiroidectomía antes de la gestación por enfermedad de Graves (DG) o con hallazgos clínicos compatibles con la enfermedad. Las gestantes con hipertiroidismo deben ser acompañadas con dosificación de TSH, T4L y T3L en la primera consulta prenatal y mensualmente. El tratamiento del hipertiroidismo se basa en el uso de propiltiouracil (PTU) y metimazol (MMZ). En el primer trimestre de gestación se debe usar el PTU debido a los efectos del MMZ en nivel embrionario. Sin embargo, el PTU deberá ser substituido por el MMZ después de este periodo por su hepatotoxicidad. Pacientes que usan PTU deben tener enzimas hepáticas monitoreadas durante el tratamiento a cada cuatro semanas, así como la función tiroidea. El diagnóstico precoz, la identificación de la etiología y el tratamiento precoz y adecuado del hipertiroidismo son importantes para evitar complicaciones como: crisis tirotóxica, aborto, hipertensión gestacional, bajo peso al nacer, restricción de crecimiento intrauterino, mortalidad al nacer, disfunción tiroidea neonatal, parto prematuro y desprendimiento prematuro de placenta.

Palabras clave: Hipertireoidismo; Embarazo; Tratamiento.

\section{Introdução}

Durante a gestação, os níveis de tiroxina total (T4) e tri-iodotironina total (T3) aumentam em decorrência da presença da gonadotrofina coriônica humana (HCG) no primeiro trimestre da gestação e do aumento da globulina ligadora de tiroxina (TBG), com consequente queda do hormônio estimulante da tireoide (TSH). A despeito dessas modificações, as frações séricas livres de tiroxina livre (T4L) e tri-iodotironina livre (T3L) permanecem nos limites da normalidade durante a gestação. Dessa forma, os baixos níveis séricos de TSH com T4L normal no início da gestação não indicam função tireoidiana anormal., ${ }^{1,23}$

A prevalência de hipertireoidismo na gestação é de aproximadamente 0,1 a $0,4 \%$, podendo se manifestar clinicamente como hipertireoidismo subclínico (ausência de sintomas), tireotoxicose e crise tireotóxica. A presença de sintomas é decorrente do efeito estimulante dos hormônios tireoidianos sobre o metabolismo e os tecidos. ${ }^{4,5}$

As principais causas de hipertireoidismo relacionadas à gestação são: hipertireoidismo gestacional mediado por HCG, doença de Graves (DG), mutação de receptor de TSH e tireoidite pós-parto, sendo os dois primeiros os de maior prevalência. ${ }^{2,3,4}$ Nos casos mal conduzidos, podem ocorrer complicações como doença hipertensiva gestacional, eclâmpsia, insuficiência cardíaca, abortamento, descolamento prematuro de pla- centa, prematuridade, baixo peso ao nascer, maior morbidade ou maior mortalidade perinatal. ${ }^{2,5,6,7}$

$\mathrm{O}$ tratamento do hipertireoidismo baseia-se no uso de drogas antitireoidianas, preferencialmente propiltiouracil e metimazol., ${ }^{2,7}$

O objetivo desta revisão é destacar a importância da identificação precoce do hipertireoidismo na gestação, possibilitando iniciar o tratamento, de preferência no primeiro trimestre, visando evitar complicações que podem acarretar graves consequências para a saúde materna e fetal.

\section{Adaptação fisiológica da gestação}

Durante a gestação, a fisiologia tireoidiana sofre algumas modificações. O aumento do estrogênio na gravidez resulta em aumento da TBG de duas a três vezes, que reduz a taxa dos hormônios tireoidianos livres séricos, estimulando o eixo hipotálamo-hipofisário-tireoidiano. ${ }^{6}$ Os níveis de T4 total e T3 total, quando comparados com valores pré-gestacionais, aumentam desde o início da gestação como resultado da elevação e redução da taxa de degradação periférica do TBG. O TBG atinge um platô com 20 semanas de gestação, mantendo-se inalterado até o final da gestação. ${ }^{1,2,3}$

Além disso, o HCG e o TSH apresentam subunidades idênticas, acarretando a estimulação do receptor de TSH pelo HCG, que estimula a liberação dos hormônios tireoidianos pela tireoide nas primeiras 8 a 14 semanas da gravidez e gera 
Tabela 1.

\begin{tabular}{l|c}
\hline \multicolumn{2}{c}{ Alvo do TSH no período gestacional. } \\
\hline Primeiro Trimestre & $0,1-2,5 \mathrm{mIU} / \mathrm{L}$ \\
\hline Segundo Trimestre & $0,2-3,0 \mathrm{mIU} / \mathrm{L}$ \\
\hline Terceiro Trimestre & $0,3-3,0 \mathrm{mIU} / \mathrm{L}$ \\
\hline
\end{tabular}

feedback negativo no eixo hipotálamo-hipofisário, com redução do TSH nessa fase gestacional. ${ }^{6} \mathrm{~A}$ função tireoidiana começa a retornar ao normal com aproximadamente 20 semanas de gestação. Em situações em que há aumento de HCG, como gestação múltipla, mola hidatiforme e hiperemese gravídica, pode haver altas concentrações séricas de T4L com supressão transitória do TSH podendo evoluir para tireotoxicose. A cada $10.000 \mathrm{mUI} / \mathrm{L}$ de HCG há queda aproximada de 0,1 $\mathrm{mUI} / \mathrm{L}$ de TSH. No segundo e terceiro trimestres há queda do HCG com consequente redução da estimulação tireoidiana e aumento do TSH. A tabela 1 refere-se aos valores do TSH nos diferentes períodos gestacionais. , $3,4^{2}$

Outro fator importante associado à fisiologia tireoidiana na gestação é o suprimento de iodo disponível à glândula tireoidiana, sendo fator importante em áreas de deficiência de iodo. Ocorre aumento na depuração do iodo pelo rim, reduzindo o iodo sérico, seguido do aumento da função tireoidiana. ${ }^{3,6}$

A glândula tireoidiana aumenta 10\% de tamanho durante a gestação e eleva sua produção em $50 \%$. A tireoglobulina sérica também aumenta no primeiro trimestre. O T4 sérico aumenta em média $0,1 \mathrm{ng} / \mathrm{dL}$ e o TSH diminui $0,1 \mathrm{mUI} / \mathrm{L} \mathrm{em}$ gestações normais. ${ }^{2}$

Gestantes eutireoideais com anticorpos antitireoidianos positivos apresentam risco aumentado de abortamento, parto pré-termo e tireoidite pós-parto. ${ }^{2}$

\section{Aspectos clínicos}

Hipertireoidismo subclínico: não há manifestações clínicas que correspondem ao hipertireoidismo laboratorial (TSH sérico suprimido ou indetectável com T3L e T4L normais). Não há evidências de que o tratamento melhore as consequências gestacionais nem que possa causar potenciais efeitos adversos ao feto., ${ }^{5,6}$ Não é associado a consequências adversas gestacionais importantes. ${ }^{8,9}$

Tireotoxicose: a ação inapropriada dos hormônios tireoidianos aumentados nos tecidos leva a tremor de extremidades, aumento da temperatura corporal, ansiedade, taquicardia, aumento pressórico principalmente do componente sistólico da pressão arterial e outros sintomas, podendo ou não estar acompanhado de estigmas da Doença de Graves.

Crise tireotóxica: há exacerbação dos sintomas simpaticomiméticos podendo evoluir para insuficiência cardíaca congestiva (ICC) e arritmia. O diagnóstico baseia-se na clínica da paciente e está associado à queda do TSH e ao aumento de T4L e T3L., ${ }^{4,6}$

\section{Diagnóstico}

O diagnóstico do hipertireoidismo é suspeitado pelos sinais e sintomas mencionados no quadro 1 e confirmado com supressão ou não detecção dos níveis séricos de TSH e elevação do T4L. Exames que utilizem iodo radioativo são contraindicados na gestação. ${ }^{2,3}$

O teste de função tireoidiana deve ser solicitado nas gestantes com hiperemese gravídica e com achados clínicos de hipertireoidismo. Outras situações em que se deve solicitar o TSH na gestação: história de abortamento e/ou parto pré-termo, anticorpos antitireoidianos positivos, diabetes mellitus tipo 1 e outras desordens autoimunes, infertilidade, história de doença familiar tireoidiana, radioterapia prévia de cabeça e pescoço, hiponatremia, anemia incompatível com os níveis encontrados na gestação. ${ }^{5,6}$

As gestantes com diagnóstico prévio de hipertireoidismo devem ser submetidas à dosagem de TSH, T4L e T3L na primeira consulta de pré-natal e mensalmente. ${ }^{5}$

O TRAb deverá ser solicitado no primeiro trimestre e entre 22 a 26 semanas de gestação quando a etiologia do hipertireoidismo for desconhecida, em pacientes tratadas com iodo radioativo ou tireoidectomia prévia por DG ou com achados clínicos compatíveis com a doença. Quando os valores são três vezes acima do limite superior da normalidade é aconselhável avaliação do bem-estar fetal e de sinais que denotem alteração tireoidiana, como bócio fetal, por ultrassonografia (USG). ${ }^{2,4,5}$ 
Quadro 1. Sinais e sintomas no hipertireoidismo. ${ }^{2,8}$

\begin{tabular}{|l|l|}
\hline \multicolumn{1}{|c|}{ SINAIS } & \multicolumn{1}{|c|}{ SINTOMAS } \\
\hline Tremor de extremidade & Palpitações \\
\hline Perda de peso & Ansiedade \\
\hline Falha de ganho ponderal a despeito de dieta adequada & Intolerância ao calor e aumento de temperatura corporal \\
\hline Taquicardia & Náuseas e vômitos \\
\hline Hipertensão & Aumento do apetite \\
\hline Tremor palpebral & Mudança de hábito intestinal \\
\hline Alargamento de tireoide ou nódulo & Irritabilidade \\
\hline
\end{tabular}

Quadro 2. Características e manejo das principais causas de hipertireoidismo. ${ }^{2,3,5,10}$

\begin{tabular}{|c|c|c|}
\hline Características & $\begin{array}{c}\text { Síndrome Hipertireoidismo } \\
\text { Gestacional }\end{array}$ & Doença de Graves \\
\hline Início & Primeira metade da gestação & Geralmente antes da gestação \\
\hline Durabilidade da doença & Transitório. Melhora com 18-20 semanas & Permanente \\
\hline $\begin{array}{l}\text { Marcadores de } \\
\text { autoimunidade }\end{array}$ & Ausente & $\begin{array}{l}\text { TRAb positivo-95\% casos } \\
\text { Anti-TPO pode ser positivo }\end{array}$ \\
\hline Etiologia & Aumento de HCG & Autoimune \\
\hline Prevalência & 1-3\% gestações & 0,1-1,0\% gestações \\
\hline Sintomatologia & $\begin{array}{l}\text { Pode cursar com tireotoxicose e hiperemese } \\
\text { gravídica. } \\
\text { Perda ou ausência de ganho ponderal }\end{array}$ & $\begin{array}{l}\text { Bócio } \\
\text { Exoftalmia } \\
\text { Tireotoxicose }\end{array}$ \\
\hline Tratamento & $\begin{array}{l}\text { Só deve ser tratada se: } \\
\text { Doença sintomática severa } \\
\text { T4L e T3L > } 50 \% \text { do valor normal para gestação } \\
\text { Suspeita de outra causa de hipertireoidismo } \\
\text { Iniciar medicações antitireoidianas. } \\
\text { Se taquicardia importante: ß-bloqueador }\end{array}$ & $\begin{array}{l}\text { PTU: 50-300 mg/dia } \\
\text { MMZ: 5-15 mg/dia } \\
\text { PPNL: 20-40 mg 6-8h } \\
\text { T4L e T3L 4/4 semanas. } \\
\text { Sintomas exacerbam no 1ํ Tri. }\end{array}$ \\
\hline Acompanhamento & Exame físico e TSH e T4L 3-4 semanas. & Melhora gradual no $2^{\circ}$ e $3 \circ$ Tri. \\
\hline
\end{tabular}

\section{Etiologia}

As principais causas de hipertireoidismo na gestação estão descritas no quadro 2. Outras causas incluem: hiperemese gravídica por aumento de HCG, hipertireoidismo trofoblástico, mutação do receptor TSHR e tireoidite pós-parto.

A hiperemese gravídica por aumento de HCG caracteriza-se por náuseas e vômitos severos no início da gestação, com perda ponderal maior que 
5\%, desidratação e cetonúria. ${ }^{2} \mathrm{O}$ tratamento é sintomático com antieméticos, hidratação e correção de distúrbios hidroeletrolíticos. ${ }^{3,5}$ Se houver manifestação clínica de tireotoxicose, administrar drogas antitireoidianas. ${ }^{3} \mathrm{O}$ aumento de HCG ocorre ainda no hipertireoidismo trofoblástico causado pela mola hidatiforme e coriocarcinoma, com alterações clínicas e ultrassonográficas típicas da doença de base e beta-HCG sérico acima do valor esperado para a idade gestacional. ${ }^{2,3}$

Deve-se ainda estar atento à possibilidade de disfunção tireoidiana no primeiro ano pósparto (tireoidite pós-parto), principal causa de tireotoxicose neste período. ${ }^{2}$ Entre dois a seis meses pós-parto, ocorre o hipertireoidismo assintomático ou sintomas hipermetabólicos leves como irritabilidade, intolerância ao calor, fadiga e palpitação. ${ }^{2} \mathrm{O}$ anti-TPO pode estar presente em alguns casos. ${ }^{3} \mathrm{O}$ tratamento está condicionado à presença de sintomas hipermetabólicos, usandose propranolol de 10 a $20 \mathrm{mg} / \mathrm{dia} \cdot{ }^{2-4} \mathrm{O}$ uso de drogas antitireoidianas é ineficaz. ${ }^{2}$ Se assintomático, repetir o TSH em 4 a 6 semanas. ${ }^{2}$ Após a paciente apresentar-se eutireoidea, dosar TSH sérico 2/2 meses até 1 ano pós-parto. ${ }^{2}$ A tireoidite pós-parto encontra-se associada com diabetes mellitus tipo 1, DG em remissão e hepatite viral crônica. ${ }^{5}$

Causas menos comuns de tireotoxicose na gestação incluem: bócio tóxico multinodular, adenoma tóxico, tireoidite aguda, hipertireoidismo iatrogênico, tumor de pituitária secretor de TSH e Struma ovarian, tumor ovariano com tecido tireoidiano em mais de $50 \%$ da massa. ${ }^{3}$

\section{Tratamento}

O propiltiouracil (PTU) e o metimazol (MMZ) inibem a produção de T4L. Além disso, o PTU também bloqueia a conversão periférica do T4 em T3, que possui maior atividade celular nos tecidos periféricos. ${ }^{10}$

O PTU deve ser usado no primeiro trimestre de gestação (devido aos efeitos do $\mathrm{MMZ}$ em nível embrionário) e em pacientes alérgicas ou com intolerância ao MMZ. Entretanto, o PTU deverá ser descontinuado após o primeiro trimestre e substituído pelo MMZ em decorrência de sua hepatotoxicidade, podendo causar necrose hepática na gestante e no concepto. As pacientes em uso de PTU devem ter as enzimas hepáticas e a função tireoidiana monitoradas a cada quatro semanas. A hepatotoxicidade induzida pelo PTU pode ser aguda, de difícil avaliação clínica e rapidamente progressiva. Se não reconhecida, pode levar à falência hepática e à morte materna., ${ }^{2,11}$

A exposição ao $M M Z$ pode causar várias malformações congênitas principalmente aplasia cutânea congênita, onfalocele, sintomas de anomalia do ducto onfalomesentérico e síndrome da embriopatia do metimazol: atresia anal ou esofageana e fácies dismórfica. O MMZ pode ser associado, em menor escala, a fístula traqueoesofágica e características dismórficas na pele. ${ }^{12}$ Todas as drogas antitireoidianas atravessam a placenta. MMZ e PTU possuem a mesma eficácia no tratamento de hipertireoidismo na gestação. .,4,5,13 $^{-13}$

A dose de PTU deve ser iniciada com 300 mg/ dia e reduzida gradativamente de acordo com o

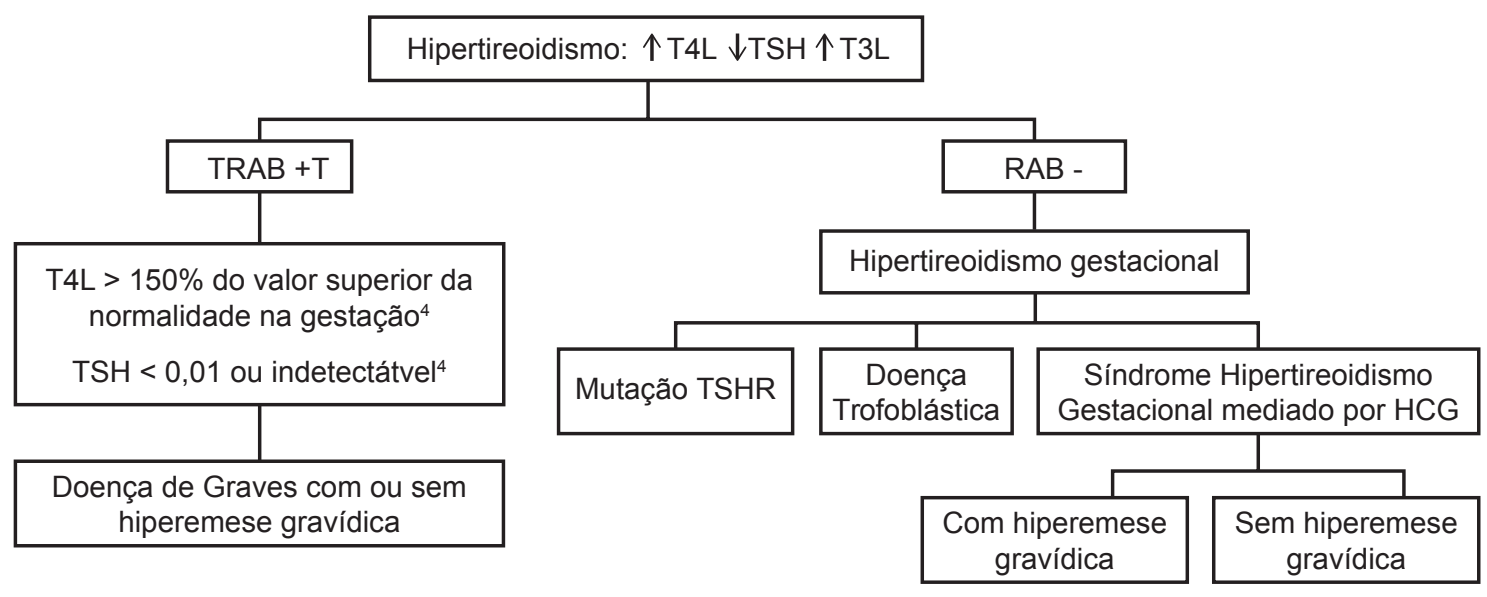

Figura 1. Diagnóstico diferencial de hipertireoidismo na gestante. 
alvo do T4L ou T4T descritos abaixo. A dose do MMZ é de 5 a 15 mg/dia (300 mg de PTU corresponde a 10-15 mg MMZ). ${ }^{4}$ Pode ser necessário associar betabloqueadores como propranolol 20-40 mg 8/8h via oral (VO).

Há ainda o risco de agranulocitose com o uso de drogas antitireoidianas, ocorrendo em $0,1 \%$ dos pacientes. Antes de iniciar a terapia medicamentosa com essas drogas deve-se solicitar leucograma de base. Pacientes com dor de garganta ou febre devem ter hemograma solicitado imediatamente. Caso ocorra agranulocitose, todas as medicações antitireoidianas devem ser imediatamente descontinuadas. ${ }^{6}$

Os agentes betabloqueadores, como o propranolol e metoprolol, podem ser usados para controle de sintomas hipermetabólicos, além de inibir a conversão de T4 em T3 nos tecidos periféricos. Na maioria dos casos, a droga pode ser descontinuada em duas a seis semanas. O tratamento por longo período de tempo pode estar associado com crescimento intrauterino restrito, bradicardia fetal e hipoglicemia neonatal., ${ }^{2,5}$

Aproximadamente de 20 a 30\% das pacientes no último trimestre da gestação têm condições clínicas de descontinuar a terapia medicamentosa na DG, exceto aquelas que se encontram com altos títulos de TRAb, em que a terapia deve ser continuada até o parto. Os sintomas frequentemente se agravam após o parto. ${ }^{2}$ Mulheres com DG antes da gestação, adequadamente tratadas e em remissão da doença, apresentam baixo risco de recorrência durante a gestação. ${ }^{4}$

MMZ de 20 a 30 mg/dia é considerada a droga antitireoidiana de primeira linha a ser utilizada na amamentação. ${ }^{2} \mathrm{O}$ PTU também pode ser usado, pois não causa alterações na função tireoidiana nem no desenvolvimento físico e psíquico do recém-nascido, porém deve ser lembrado o risco de hepatotoxicidade. ${ }^{14}$

A tireoidectomia deve ser considerada em caso de alergia, contraindicações às drogas antitireoidianas, em pacientes que requerem altas doses das medicações (MMZ30 mg/dia e PTU 450 $\mathrm{mg} / \mathrm{dia}$ ) ou quando não há resposta ao tratamento medicamentoso. O segundo trimestre é o melhor momento para realização da cirurgia, pois esta é fator de risco para parto prematuro (risco entre $4,5$ e $5,5 \%)^{4,5,14}$

O acompanhamento do tratamento deve ser baseado na dosagem sérica mensal dos hormônios tireoidianos. ${ }^{4} \mathrm{O}$ T4L deve permanecer no limite superior da normalidade e o T4T até 1,5 vezes do valor normal para gestantes. O TSH não é solicitado na monitorização do tratamento porque se mantém baixo. A melhora dos sintomas ocorre em 3 a 4 semanas de tratamento, mas a resposta plena ocorre por volta de oito semanas pós-tratamento. Pode-se avaliar a suspensão da medicação antitireoidiana após a paciente manter-se eutireoideia próxima ao termo. ${ }^{5,6,15}$

Fetos de mães em uso de drogas antitireoidianas devem ser avaliados quanto aos sinais de hipotireoidismo por exame clínico para crescimento e cardiotocografia para avaliação de bradicardia fetal. Aproximadamente 10\% dos expostos a estas drogas desenvolverão hipotireoidismo fetal e neonatal. A USG deverá ser solicitada para avaliação de biometria fetal e avaliação de sinais de bócio fetal: presença de massa paratraqueal simétrica, hiperextensão do pescoço e polidramnia., ${ }^{6,14}$

Caso a mulher seja exposta à terapia ablativa, deve manter-se em uso de contraceptivo seguro por, no mínimo, seis meses. Este tratamento é contraindicado durante a gestação e lactação devido ao seu efeito teratogênico. ${ }^{6}$

\section{Complicações}

O controle inadequado da tireotoxicose é responsável pelas seguintes complicações: abortamento, hipertensão gestacional, baixo peso ao nascer, restrição de crescimento intrauterino, natimortalidade, tempestade tireotóxica, disfunção tireoidiana neonatal, parto prematuro e descolamento prematuro de placenta. ${ }^{2,5,6,7,11}$

\section{Crise tireotóxica}

É evento raro na gestação. O controle inadequado do hipertireoidismo pode evoluir para tempestade (crise) tireotóxica com aumento da mortalidade materno-fetal. ${ }^{16}$

O diagnóstico é clínico com atividade adrenérgica exacerbada, taquicardia desproporcional à idade gestacional, aumento da temperatura corporal, agitação ou confusão mental, podendo evoluir para ICC e arritmia cardíaca. ${ }^{6}$ Pode ocorrer ainda delirium, psicose, convulsão, diarreia e evolução para estado comatoso..$^{10}$ Durante a crise 
Atividade adrenérgica exarcebada, taquicardia desproporcional à idade gestacional, aumento da temperatura corporal, agitação ou confusão mental. Sinais e sintomas de ICC.

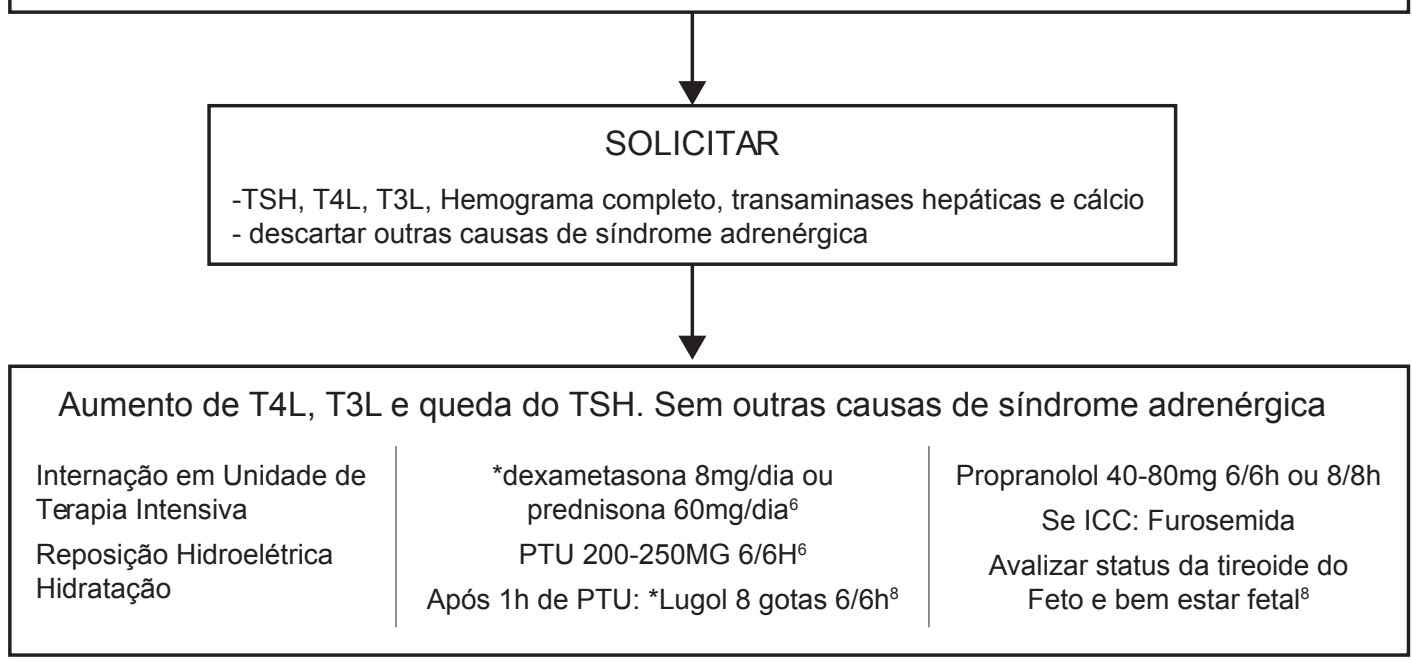

*Iodo e o corticoide podem ser descontinuados após a estabilização clínica.

Figura 2. Conduta mediante diagnóstico de crise tireotóxica ${ }^{6,8}$

tireotóxica, pode haver aumento de transaminases hepáticas, leucocitose e hipercalcemia. Além disso, deve-se atentar para fatores desencadeantes como infecções, cirurgias, complicações clínicas, pré-eclâmpsia e parto. O tratamento deverá ser em Unidade de Terapia Intensiva. ${ }^{6,16}$

A crise tireotóxica não indica interrupção da gestação. O parto por demais indicações obstétricas não deverá ocorrer antes da compensação clínica da paciente, visto que o parto cesáreo ou via vaginal podem exacerbar a crise tireotóxica. ${ }^{16}$

\section{Conclusão}

O médico obstetra deve estar atento às manifestações clínicas compatíveis com hipertireoidismo, a fim de diagnosticar e tratar precocemente as pacientes, reduzindo as complicações obstétricas e a morbimortalidade materno-fetal relacionada à essa condição clínica.

\section{Referências}

1. Mestman JH. Thyroid and parathyroid diseases in pregnancy. In: Gabbe SG, Niebyl JR, Simpson JL, Landon MB, Galan HL, Jauniaux ERM, et al. Obstetrics normal and problema pregnancies. 6 ed. Philadelphia; 2012. p.922-53.

2. Stagnaro-Green A, Abalovich M, Alexander E,
Azizi F, Mestman J, Negro R, et al. Guidelines of the American Thyroid Association for the diagnosis and management of thyroid disease during pregnancy and postpartum. Thyroid. 2011;21:1081-125. http:// dx.doi.org/10.1089/thy.2011.0087.

3. Albaar MT, Adam JM. Gestational transient thyrotoxicosis. Acta Med Indones. 2009;41(2):99-104.

4. Bahn RS, Burch HB, Cooper DS, Garber JR, Greenlee MC, Klein I, et al. Hyperthyroidism and other causes of thyrotoxicosis: management guidelines of the American Thyroid Association and American Association of Clinical Endocrinologists. Thyroid. 2011;21(6):593-646.http://dx.doi.org/10.1089/ thy.2010.0417.

5. Groot LD, Abalovich M, Alexander EK, Amino N, Barbour L, Cobin RH, et al. Management of thyroid dysfunction during pregnancy and postpartum: An Endocrine Society clinical practice guideline. J Clin Endocrinol Metab. 2012;97(8):2543-65. http://dx.doi. org/10.1210/jc.2011-2803.

6. Fitzpatrick DL, Russell MA. Diagnosis and management of thyroid disease in pregnancy. Obstet Gynecol Clin North Am. 2010;37(2):173-93. http:// dx.doi.org/10.1016/j.ogc.2010.02.007.

7. Inoue M, Arata M, Koren G, Ito S. Hyperthyroidism during pregnancy. Can Fam Physician. 2009;55(7):701-3.

8. Ohashi M, Furukawa S, Michikata K, Kai K, Sameshima H, Ikenoue T. Risk-based screening for thyroid dysfunction during pregnancy. J Pregnancy. 2013; 2013:619718. http://dx.doi. 
org/10.1155/2013/619718.

9. Casey BM, Dashe JS, Wells E, McIntire DD, Leveno KJ, Cunnngham FG. Subclinical hyperthyroidism and pregnancy outcomes. Obstet Gynecol. 2006;107:337-41.

10. Landenson P, Kim M. Thyroid. In: Lee G, Ausiello D. Cecil Textbook of Internal Medicine. 23 ed. 2008. p.1698-717.

11. Chen CH, Xirasagar S, Lin CC, Wang LH, Kou YR, Lin HC. Risk of adverse perinatal outcomes with antithyroid treatment during pregnancy: a nation wide population-based study. BJOG. 2011;118(11):136573. http://dx.doi.org/10.1111/j.1471-0528.2011.03019.x.

12. Yoshihara A, Noh J, Yamaguchi T, Ohye H, Sato S, Sekiya K, et al. Treatment of Graves' disease with antithyroid drugs in the first trimester of pregnancy and the prevalence of congenital malformation. J Clin Endocrinol Metab. 2012; 97(7):2396-403. http://dx.doi.org/10.1210/jc.2011-2860

13. Glinoer D, Cooper DS. The propylthiouracil dilemma. Curr Opin Endocrinol Diabetes Obes. 2012;19(5):402-7. http://dx.doi.org/10.1097/MED.0b013e3283565b49

14. Alamdari S, Azizi F, Delshad H, Sarvghadi F, Amouzegar A, Mehran L. Management of hyperthyroidism in pregnancy: comparison of recommendations of American Thyroid Association and Endocrine Society. J Thyroid Res. 2013;2013:878467. http://dx.doi. org/10.1155/2013/878467

15. British Thyroid Foundation. UK guidelines for the use of thyroid function tests. UnitedKingdom; 2006.
16. Khoo CM, Lee KO. Endocrine emergencies in pregnancy. Best Pract Res Clin Obstet Gynaecol. 2013;27(6):885-91. http://dx.doi.org/10.1016/j. bpobgyn.2013.08.005

\section{Bárbara B.G. R. Bártholo}

Programa de Pós-graduação em Ciências Médicas. Faculdade de Ciências Médicas. Universidade do Estado do Rio de Janeiro. Rio de Janeiro, RJ, Brasil.

\section{Denise L. M. Monteiro}

Disciplina de Obstetrícia. Departamento de Ginecologia e Obstetrícia. Centro Universitário Serra dos Órgãos (UNIFESO). Teresópolis, RJ, Brasil.

Disciplina de Obstetrícia. Departamento de Ginecologia e Obstetrícia. Faculdade de Ciências Médicas da Universidade do Estado do Rio de Janeiro. Rio de Janeiro, RJ, Brasil.

\section{Alexandre J. B. Trajano}

Departamento de Ginecologia e Obstetrícia. Faculdade de Ciências Médicas. Universidade do Estado do Rio de Janeiro. Rio de Janeiro, RJ, Brasil.

Departamento de Ginecologia e Obstetrícia. Faculdade de Medicina. Universidade Unigranrio. Rio de Janeiro, RJ, Brasil. 